\title{
India as "The Oral Cancer Capital of the World": The Rising Burden of Oral Malignancies across the Nation
}

\author{
Hindol Das ${ }^{1}$, Shilpa Motghare ${ }^{2}$ \\ ${ }^{1}$ B.D.S., Private Practitioner \\ ${ }^{2}$ Tutor, Dept. of Pathology, Govt. Medical College, Shivpuri \\ Corresponding Author: Hindol Das
}

\begin{abstract}
India is a global epicenter of oral cancer patients and the magnitude of the problem is ever increasing day by day. There is excess burden of oral malignancies all over the country and the risk factors associated with the disease are at its peak. Addressing the disease which is quite like an epidemic is a great challenge and major public health issue in India. This review paper discusses the burden of the disease, its top risk factors in India including the use of tobacco, alcohol, areca nut and HPV infections; and methods for prevention and control of oral cancer in India.
\end{abstract}

Keywords - Oral cancer, Burden, Risk factors, Tobacco, Alcohol, Areca nut, HPV, Prevention

\section{INTRODUCTION}

India has the most number of oral cancer cases than any other nation worldwide and recently came to be known as "The Oral Cancer Capital of the World". There has been an exponential rise in oral cancer cases throughout the country because of the lack of awareness and knowledge of the common people. This may be attributed to the high use of tobacco, alcohol, chewing of betel quid and areca nut products to name a few. The massive burden of oral cancer in India is a great public health challenge ${ }^{(1)}$. Most of the oral cancer cases in India are detected at an advanced stage when the outcome of treatment is very poor and simultaneously the cost for treatment becomes expensive for the patient and their families. Unaffordable treatment option at later stages of oral cancer is highly prevalent in India. In addition, most rural areas have inadequate access to health services and lack of trained clinicians which makes it even tougher to screen oral cancers at an early stage. Early detection and screening of oral cancers in these rural areas even at the stage of oral potentially malignant disorders (OPMD) could restrict transformation to malignant conditions such as oral squamous cell carcinomas (OSCC) (2).

Oral Cancer includes cancer of the lip, oral cavity, nasopharynx and pharynx. Squamous cell carcinoma (SCC) is the most common type of oral cancer contributing to around $84 \%$ to $97 \%$ of the cases. Oral cancer results from preclinical stage of oral potentially malignant disorders (OPMD) such as leukoplakia, erythroplakia, lichen planus, oral submucous fibrosis, etc. South Central Asia has a relatively higher burden of oral cancer. In India, oral cancer is directly associated with people from low socioeconomic status due to larger risk factor exposures of which the use of tobacco and alcohol remain as the major risk factors accompanied by the extensive use of areca nut and betel quid, viral infections (HPV, HSV-1), poor oral hygiene, nutritional deficiency, etc. ${ }^{(3)}$ India has become a global hub of oral cancer patients with poor 

the nation.

prognosis and high mortality rate of the disease.

\section{Oral Cancer Burden in India}

India contributes one-third of the total burden of oral cancer cases in the world. Oral cancer accounts $30 \%$ of all cancers reported in India. It ranks among the top three cancers in the country. In India, Oral cancer ranks first among all cancers in men and second among all cancers in women as depicted in Table $1^{(4)}$. Sex ratio indicates 2:1 male predominance. In males, oral cancer and lung cancer contributes greater than $25 \%$ cancer deaths in India and in females, breast cancer and oral cancer contributes $25 \%$ cancers in India. The list of top five cancers in both sexes contributes $47.2 \%$ of total cancers in India. These cancers can be prevented if screened, detected and treated at an early stage and can remarkably reduce the mortality rate ${ }^{(5)}$.

Table 1: List of top five cancers in India among men and women

\begin{tabular}{|l|l|l|}
\hline Rank & Men & Women \\
\hline 1st & Lip, Oral cavity & Breast \\
\hline 2nd & Lung & Lip, Oral cavity \\
\hline 3rd & Stomach & Cervix \\
\hline 4th & Colorectal & Lung \\
\hline 5th & Esophagus & Gastric \\
\hline
\end{tabular}

Globally, $57.5 \%$ of oral cancer cases occur in Asia, particularly in India. The average age of oral cancer in India is 50 years. 60 to $80 \%$ of patients in India report with advanced disease as compared to $40 \%$ in developed countries. There is poor survival rate of 5 years (overall) mainly due to large proportion of advanced cases. Survival rate in India is only $27 \%$ for patient with advanced stage of oral cancer. Since cancer registration is not mandatory in India, a large number of cases go unrecorded or are lost to follow-up and the true incidence and deaths might go higher than the normal figures ${ }^{(6)}$.

\section{Risk Factors at the Peak Tobacco}

It is the most significant modifiable risk factor for oral cancer. $80-90 \%$ of oral cancer cases is directly linked to the use of tobacco. Greater than $80 \%$ of the total tobacco users in the world reside in low and middle-income countries. According to reports of National Family and Health Survey, about one in ten adults aged 15 to 49 years use some form of tobacco in India (7). The use of tobacco is higher among males and more common in uneducated, backward and economically weak sections of the society. There is inter-state variation in the prevalence and use of different forms of tobacco in India. The north-eastern states has the highest prevalence of tobacco use in India with Tripura at the top as per the report of the 2016-17 Global Adult Tobacco Survey (GATS-2) as given in Figure ${ }^{\left({ }^{(8)}\right.}$.

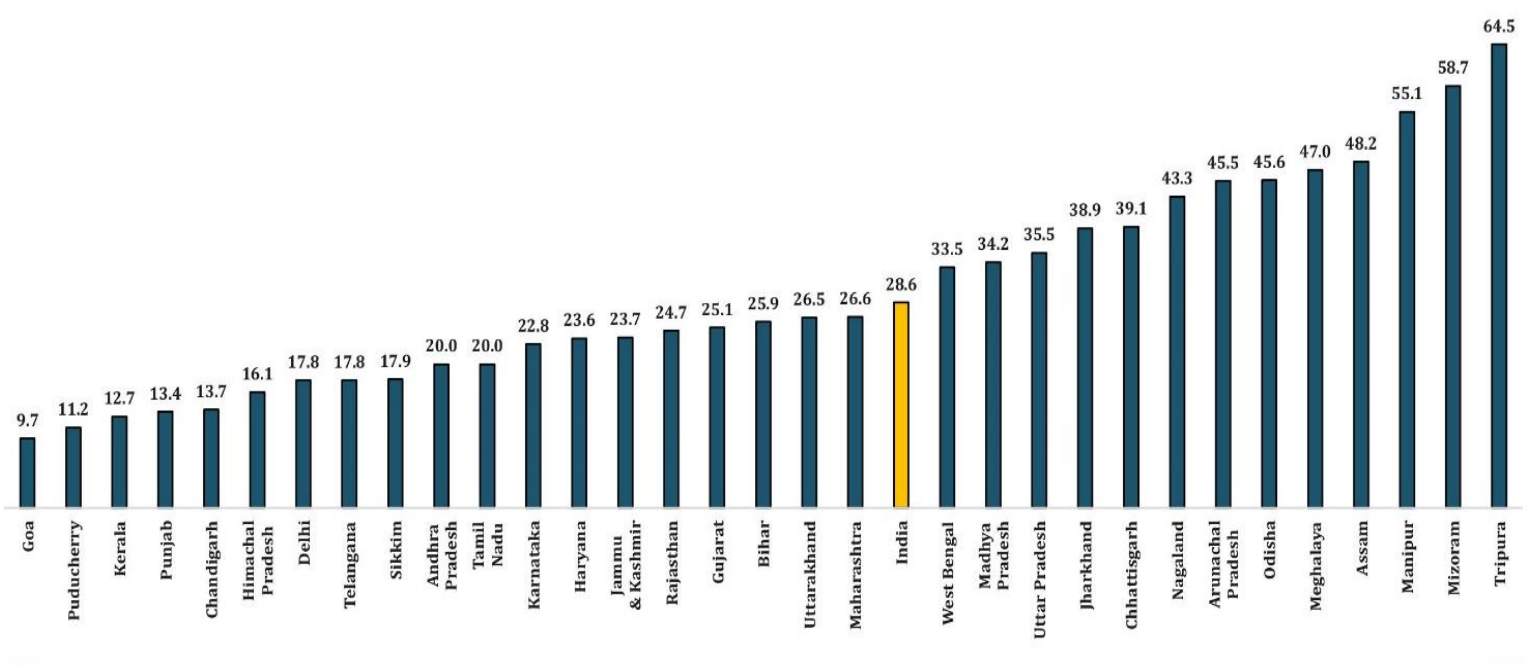

Figure 1: Prevalence of Tobacco use (both smoking \& smokeless) among states/UTs in India, GATS-2 

the nation.

International Agency for Research on Cancer (IARC) has classified Tobacco as group I carcinogen. The different types of tobacco available in India are classified according to the constituents, method of processing and mode of use. The two most popular forms include smoking tobacco (combustible) and smokeless tobacco (noncombustible). Approximately seventy species of tobacco (Nicotiana) exists all over the world. The common species used in tobacco products include $N$. tabacum, $N$. rustica, $N$. glauca. In India, smoked tobacco products are made from $N$. tabacum and smokeless tobacco products are made from $N$. rustica with or without $N$. tabacum ${ }^{(9)}$. None of the type of tobacco products are considered safe and both the types of tobacco can cause oral cancers. Different types of smoking and smokeless tobacco products in India are illustrated in Table 2. The epidemiology of tobacco use in India is quite unique as there are more smokeless tobacco users than smokers. Percentage distribution of adults by tobacco use in India as of Global Adult Tobacco Survey (GATS2 ) is enumerated in Figure 2. The prevalence of smoking tobacco and smokeless tobacco use among states in India is given in Figure 3.1 \& Figure 3.2 respectively ${ }^{(10)}$.

In India, the most frequently consumed tobacco products are khaini and bidi accounting for $11 \%$ and $8 \%$ respectively (Global Adult Tobacco Survey2). Studies conducted by Rahman et al found that bidi smokers in India have three times increased risk for oral cancer when compared to that of nonsmokers, while on the contrary no notable risk pattern was found for cigarette smokers. This may be due to poor combustibility and the levels of nicotine in bidi which is higher than that in cigarette. Besides, another study from Kerala found that the risk of oral cancer is nearly five times higher for tobacco chewers in comparison to non-chewers. Smokeless tobacco products contain the most toxic and harmful compound known as tobaccospecific nitrosamines (TSNAs) and their quantity is directly linked with risk of oral cancer ${ }^{(11)}$. The carcinogenic potential of tobacco and its constituents is demonstrated in Table 3. Among the smokeless tobacco (SLT) products, dry snuff have higher risk for oral cancer whereas moist snuff along with chewing tobacco have a very low relative risk while the other types of smokeless tobacco is of intermediate risk for oral cancer. The trend of using smokeless tobacco in India is increasing not only among men but also among young individuals, women and children leading to alarming numbers of oral cancer cases in this subgroups as well and therefore the large population must be alert on this public health issue ${ }^{(12)}$.

Table 2: Different types of Smoking and Smokeless tobacco products in India

\begin{tabular}{|l|l|}
\hline $\begin{array}{l}\text { Smoking } \\
\text { Tobacco }\end{array}$ & Smokeless Tobacco (SLT) \\
\hline $\begin{array}{l}\text { Bidis, } \\
\text { Cigarettes }\end{array}$ & $\begin{array}{l}\text { Chewable- Khaini, Gutka, Betel quid with } \\
\text { tobacco, Mawa, Tobacco leaf, Zarda, Kiwam, } \\
\text { Mainpuri, Dohra }\end{array}$ \\
\hline Hookah & $\begin{array}{l}\text { Snuffing local application- Gul, Gudhaku, } \\
\text { Creamy snuff, Mishri, Tapkir/Bajjar, Khaini }\end{array}$ \\
\hline Chilum & Suckable- Mishri, Khaini \\
\hline Pipe & Gargle- Tuibur/ Hidakpha \\
\hline Cigar & Nasal Use- Tapkir \\
\hline
\end{tabular}

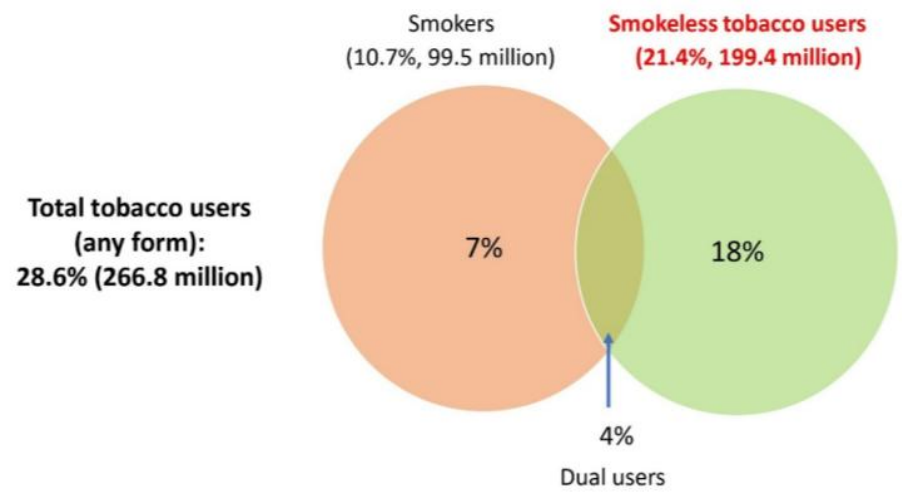

Figure 2: Percentage distribution of adults by tobacco use in India (GATS-2) 
Hindol Das et.al. India as "the oral cancer capital of the world": the rising burden of oral malignancies across the nation.

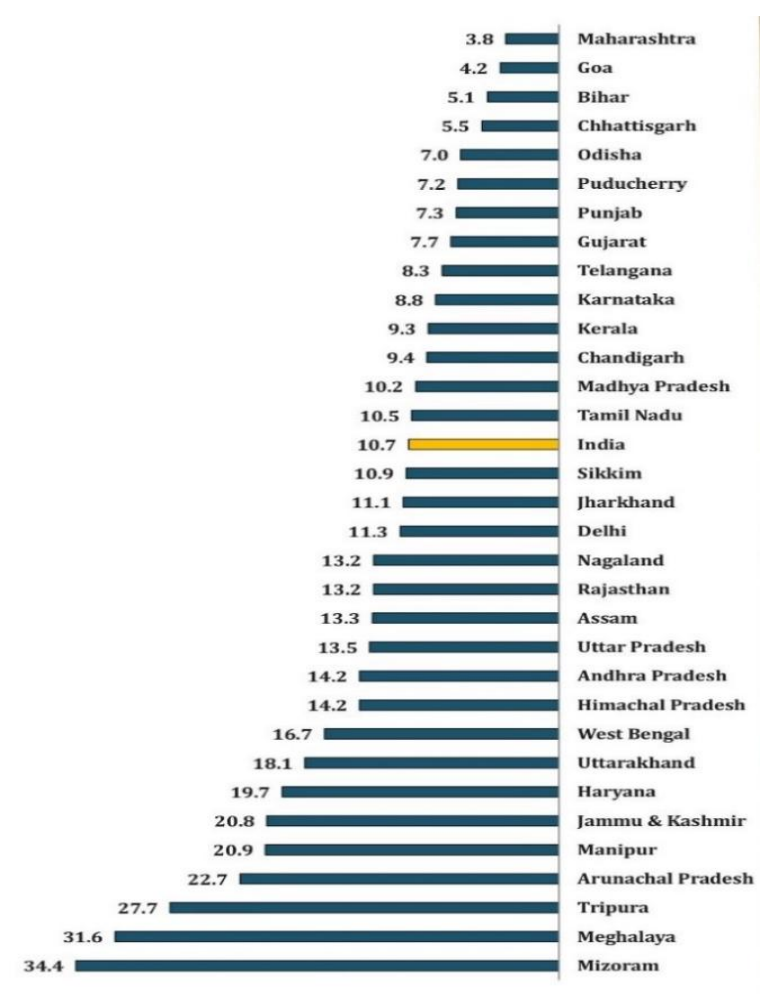

Figure 3.1: Prevalence of Smoking tobacco use among states/ UTs in India (Adapted from GATS India 2016-17)

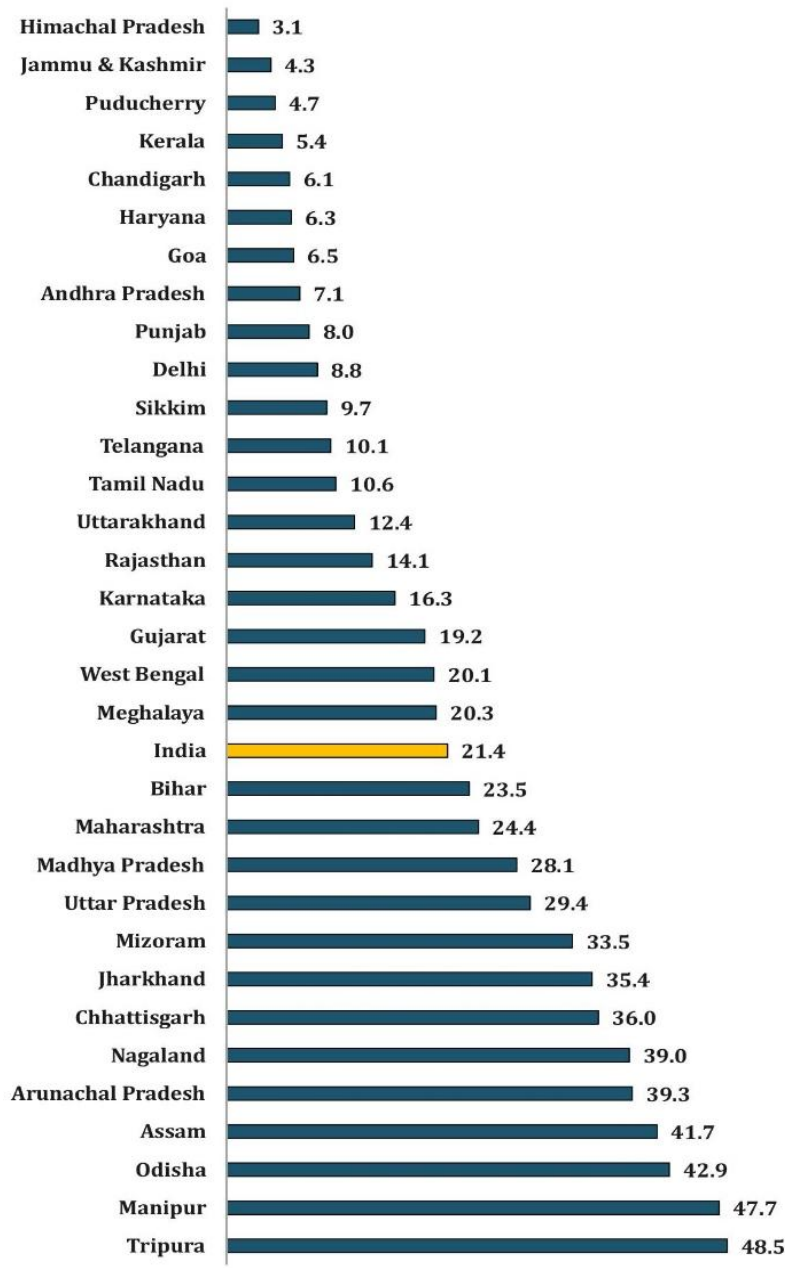

Figure 3.2: Prevalence of Smokeless tobacco use among states/ UTs in India (Adapted from GATS India 2016-17)
Table 3: The carcinogenic potential of tobacco and its constituents

\begin{tabular}{|l|l|}
\hline Constituents & Detrimental Effects \\
\hline $\begin{array}{l}\text { Poly aromatic } \\
\text { hydrocarbon }\end{array}$ & Carcinogenesis. \\
\hline $\begin{array}{l}\text { Nicotine } \\
\text { Nitrosamine }\end{array}$ & Potential carcinogenic agents. \\
\hline Phenol & $\begin{array}{l}\text { Ganglionic stimulation and depressions, } \\
\text { Tumor promotion. }\end{array}$ \\
\hline Benzopyrene & Tumor promotion, Irritation. \\
\hline Carbon mono oxide & Impaired oxygen transport and repair. \\
\hline Formaldehyde & Toxicity to cilia and irritation. \\
\hline
\end{tabular}

\section{Alcohol}

Independently alcohol has 4\% attributable risk for oral cancer, whereas tobacco alone contributes $33 \%$ of risk and their combined risk (alcohol + tobacco) is reported to be $35 \%$ which indicates that both alcohol and tobacco together has a synergistic effect in development of oral cancer ${ }^{(13)}$. It promotes greater permeability and increased penetration of carcinogens through the oral mucosa. Alcohol in particular increases the risk for hypopharyngeal cancer. According to a study by Znaor et al, the combined effects of tobacco smoking, chewing and alcohol consumption was found to induce twenty four times increased risk for oral, pharyngeal and esophageal cancers. Several studies have shown that there exists a strong positive connection between use of alcohol and smoking. Alcohol dependent individuals are three times more likely to be smokers than those in the general population and tobacco dependent individuals are four times more likely to be alcoholic than those in the general population. In India, alcohol associated tobacco use is very common among the masses and thus is a remarkable risk factor for oral cancer ${ }^{(14)}$.

\section{Areca Nut}

Areca nut is the seed of the areca palm (areca catechu) found in South-East Asia and East Africa. The use of areca nut is an independent risk factor for development of oral malignancy. Many studies have reported an increased risk for oral cancers in "areca nut users alone". It has been considered as type I human carcinogen by IARC (International Agency for Research on Cancer). Areca nut is the fourth most 
frequently used psychoactive substance across the globe following caffeine, nicotine and alcohol. There are 600 million people all over the world who use areca nut and its use is popular in Asian countries like India and Taiwan and also quite common in parts of Sri Lanka, Maldives, Bangladesh, Myanmar, Thailand, Indonesia, Malaysia, Cambodia, Vietnam, Philippines, Laos, China and many islands in South Pacific. In India, percentage of adults using different non-tobacco products including areca nut is given in Figure 4. The addiction of areca nut in India is high among both males and females and the user distribution is elevated in states like Tripura (22.6\%), Tamil Nadu (19.1\%), Maharashtra (17\%) and Puducherry (15.1\%). Few commercially available areca nut products in India include Kottapak/Chali, Kalipak, Scented Supari (North India), Bura Tamul (North-East India), Mawa (Gujarat), Neetadaka, Tamol, Parcha, etc. ${ }^{(15)}$.

Areca nut is consumed in ripe or unripe form and may be dried, baked or roasted; cut into slices, crushed or taken as a whole. It is a common constituent of a wide variety of chewed products available in India. Areca nut may or may not be mixed with tobacco and also may be wrapped in betel leaves (paan) - leaf of the betel plant to have variety of preparations. The name "betel nut" is often used instead of "areca nut", but it is wrong since betel plants do not contain fruits but only have leaves (betel leaves). Chewing of betel quid, i.e. paan, areca nut and tobacco combined together is very popular in India, however the use of betel quid have 8 to 15 -fold increased risk for oral cancer compared to that of 1-4 times associated with using the quid alone without tobacco ${ }^{(16)}$. Other preparations of areca nut include gutka (a combination of tobacco and areca nut mixed with lime and flavouring agents) and paan masala (without tobacco) sold as mouth fresheners, digestive aids and as stimulants. It must be noted that all forms of areca nut can induce oral carcinogenesis and its use can lead to oral potentially malignant disorders (OPMD) - most common being oral submucous fibrosis (OSMF). The contents of areca nut that have been proven carcinogenic are Tannins, Polyphenols (safrole, hydroxychavicol and catechins) and Alkaloids (guvacine, arecaidine, guvacoline and arecoline). Chewing of areca nut produces reactive oxygen species (ROS) that induces inflammation in the oral mucosa, it also causes genotoxicity, growth arrest and apoptosis of the cells, it may bring about autophagy and inhibit tumor suppressors, it also causes tissue hypoxia and help in carcinogenesis, at the molecular level it also facilitates cell motility and epithelialmesenchymal transition and promotes chemo-radioresistance all of which leading to oral malignant transformation (17).

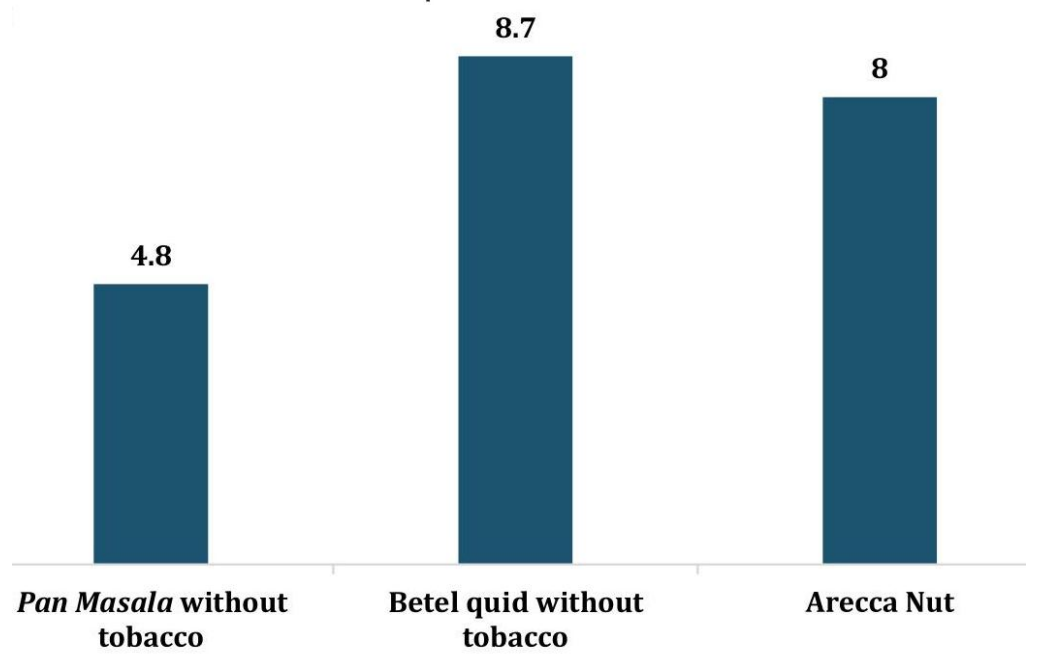

Figure 4: Percentage of adults using different non-tobacco products including areca nut in India, GATS-2 


\section{Human Papilloma Virus (HPV) infection HPV types 2, 3, 6, 11, 13, 16, 18, 31,} $33,35,52$ and 57 are associated with malignant lesions of the oral cavity. The high-risk types are 16, 18, 31 and 33 that are seen in almost $99 \%$ of HPV linked oral cancers. Relation between a subtype of HPV 16 and oropharyngeal cancers has been reported by IARC (International Agency for Research on Cancer). There is a strong correlation between cancer of the tonsil and oropharynx to that of HPV. Several studies have shown that HPV may be significantly associated in the etiology of oral cancer. According to a good deal of research, a link between HPV 16 and 18 with oropharyngeal squamous cell carcinomas (SSCs) in individuals with no apparent history of longterm use of tobacco and alcohol has been established. Such HPV associated oropharyngeal SSCs have a favourable outcome when compared to other head and neck SSCs and they also have increased sensitivity to radiotherapy ${ }^{(18)}$.

HPV related oral SSCs is more common in India than other nations. In India, the incidence of HPV in oral carcinomas may be noticed as high as in $74 \%$ of the cases. According to a study done by Balaram et al, it was found that the prevalence of oral SSCs cases recognized as HPV-positive was $67 \%$ in southern India, $34 \%$ in eastern India and $15 \%$ in western India. It is more frequently seen in younger patients less than 50 years of age and common sites include the base of the tongue and tonsil region. Probable connection exists with sexual practice such as oral sex, frequent sexual indulgence without use of barriers, sex with multiple partners, sex at an earlier age, etc. ${ }^{(19)}$.

\section{Other factors}

Apart from tobacco, alcohol, areca nut and viral infections; oral cancer may also be caused due to chronic irritation or trauma, nutritional deficiency, genetic predisposition, candidal infection, poor oral hygiene, mouthwashes, immunosuppression, exposure to sun/ ultraviolet radiations and occupational risk. Out of all these, poor oral hygiene is a prominent risk factor in India. According to a study, poor oral hygiene was seen in $85 \%$ of oral cancer patients. Moreover, attributable risk for oral cancer in India due to poor oral hygiene is $32 \%$ in males whereas $64 \%$ in females ${ }^{(20)}$. Long term use of denture over the years is also common among aged individuals in India that leads to oral cancers.

\section{Approaches for Prevention and Control of Oral Cancer in India Early Diagnosis and Screening}

In India, oral cancer is detected mostly at advanced stages when the prognosis becomes poor and people are left with no choice for treatment. However, if they can be screened and diagnosed at an early stage, it would ensure improved treatment outcome, better curing rates and will also bring down the treatment cost so that it can be affordable and at the same time will drastically reduce the morbidity rate. In country like India, cost-effective oral cancer screening must be carried out by trained clinicians, health care professionals and dental surgeons in large and high-risk population (people using tobacco, alcohol, areca nut or with poor oral hygiene) as opportunistic screenings or population-wide screenings. Studies have proved that early detection and screening of oral cancer can significantly reduce the mortality due to the disease ${ }^{(21)}$

Initiative and intervention must also be taken by the government in the form of Oral Cancer Control Policy such as a well executed and operated National Oral Cancer Control Programme and National Oral Cancer Screening Programmes can be proposed. A large workforce of healthcare individuals from the country can be employed to perform oral visual examination of the diverse population to detect the clinico-diagnostic features of oral potentially malignant disorders (OPMD) and oral cancers as well as assessment of risk factors. It can be a cost-effective 
screening tool to rule out oral malignancies at early stages ${ }^{(22)}$. Besides, oral health examination should be made mandatory part of systemic health examination in all patients particularly subjects with history of habits. Just similar to the western countries, government must also take action to make routine oral health checkup compulsory every six months in India.

\section{Eliminate Risk Factors}

Quitting habits of the use of tobacco, alcohol and areca nut- the major risk factors in India will put an end to oral cancer. The most tactful and cost effective strategy include modification of risk factors with most important being tobacco control. Utmost priority should be given in controlling smokeless tobacco use which is pretty high in India. Early detection and screening would not help prevent oral cancers if consumption of tobacco remains high. Efforts must include- increasing taxes on all types of tobacco and alcohol products, strict pictorial warnings on the labels of tobacco and alcohol products, legislative measures on youth access to tobacco, initiate tobacco control in schools, prohibit tobacco containing food products like gutka, tobacco dentifrices, etc. under Food Safety and Standards Act of India, incorporate a National Alcohol Control Policy to lessen promotion, advertisement, sale, manufacture and use of alcoholic products; control use of areca nut products and their distribution, etc. (23)

Government has initiated National Tobacco Control Programme (NTCP) in India with an aim to create awareness, decrease production and supply of tobacco products and help individuals quit tobacco. NTCP works through the central, state and district level with tobacco cessation services for the common people. National Tobacco Control Cell (NTCC) under Ministry of Health and Family Welfare (MoHFW) monitors and evaluates activities of the NTCP. Private organizations like Indian Dental Association (IDA) with a slogan "tobacco-free India" has established network of TII (Tobacco Intervention Initiative) centres for tobacco control in India and IDA has also launched OCF (Oral Cancer Foundation) which aims 'cancer-free India' is quite appreciable. There is multiple Spot Prevent Oral Cancer Trauma (SPOT) centers under the OCF. An online National Oral Cancer Registry exclusively for dental clinics and hospitals has also been set up by IDA $^{(24)}$.

\section{Public Awareness and Promotion of Oral Health}

Oral cancer can be detected early by increasing awareness in the community and training the masses for self-oral examination of the mouth, which can be a simple yet effective tool in detecting any abnormalities of the oral cavity by the patient itself ${ }^{(25)}$. Role of HPV in oral cancer can be explained to people in socially acceptable communities by promoting health programmes and encouraging safe sexual practices. Intervention can be made in the community to raise public awareness regarding the use of tobacco and alcohol; and habit counselling may be done for the high-risk individuals. Public health educational campaigns regarding promotion of good oral hygiene and maintenance of oral health is the key to prevention of oral cancer and other diseases of the oral cavity in country like India where there is immense lack of knowledge regarding oral health. The month of April is considered as "Oral Cancer Awareness Month" and this can be the right time of the year for dentists and clinicians to highlight the dangers of oral cancer to the public and motivate them to get their mouth screened for any abnormalities ${ }^{(26)}$.

\section{CONCLUSION}

More than five persons die from oral cancer every hour daily in India and nearly same numbers of individuals die from malignancies of the oropharynx and hypopharynx. It is high time to understand the growing burden of oral cancer in India and immediate intervention is required to 
decrease the incidence rate, improve the quality of life and reduce mortality across the country. Risk factors that are on the rise must be controlled effectively and efficiently by the central and state governments with strict regulations. Oral cancer control in India can be reached by a simple strategy of common risk factor approach by integrating oral health with systemic health. A lot more research and policy initiatives is required to bring down the oral cancer rate in the country.

\section{Acknowledgement: None}

\section{Conflict of Interest: None}

\section{Source of Funding: None}

\section{REFERENCES}

1. Gupta B, Ariyawardana A, Johnson N. Oral cancer in India continues in epidemic proportions: evidence base and policy initiatives. International Dental Journal. 2013;63(1):12-25.

2. Sankaranarayanan R. Oral cancer in India: An epidemiologic and clinical review. Oral Surgery, Oral Medicine, Oral Pathology. 1990;69(3):325-330.

3. Borse V, Konwar A, Buragohain P. Oral cancer diagnosis and perspectives in India. Sensors International. 2020;1:100046.

4. Coelho K. Challenges of the Oral Cancer Burden in India. Journal of Cancer Epidemiology. 2012;2012:1-17.

5. Mathew B. P3.88. An epidemic of oral cancer predicted by 2020 in India. Oral Oncology Supplement. 2009;3(1):230.

6. Swaminathan R, Rama R, Shanta V. Lack of active follow-up of cancer patients in Chennai, India: implications for populationbased survival estimates. Bull World Health Organ 2008 86: 509-515.

7. Pradhan M, Patel S, Prusty R. Pattern and Predictors of Tobacco Use in India: Evidence from National Family Health Survey (2015-2016). Journal of Health Management. 2019;21(4):510-524.

8. Ruhil R. Sociodemographic Determinants of Tobacco Use in India: Risks of Risk FactorAn Analysis of Global Adult Tobacco Survey India 2016-2017. SAGE Open. 2019;9(2):215824401984244.
9. Subramanian SV, Nandy S, Kelly M et al. Patterns and distributionof tobacco consumption in India: cross-sectional multilevelevidence from the 1998-9 national family health survey. BMJ 2004 328: 801-806.

10. Mini G, Thankappan K. Switching to smokeless tobacco, the most common smoking cessation method: results from the Global Adult Tobacco Survey, India. Public Health. 2016;136:172-174.

11. Jayalekshmi PA, Gangadharan P, Akiba S et al. Oral cavity cancer risk in relation to tobacco chewing and bidi smoking among men in Karunagappally, Kerala, India: Karunagappally cohort study. Cancer Sci 2010 102: 460-467.

12. Schulz M, Reichart PA, Ramseier CA et al. Smokeless tobacco:a new risk factor for oral health? A review Schweiz Monatsschr Zahnmed 2009 119: 1095-1109.

13. Hashibe M, Brennan P, Chuang SC et al. Interaction between tobacco and alcohol use and the risk of head and neck cancer: pooled analysis in the International Head and Neck Cancer Epidemiology Consortium. Cancer Epidemiol Biomarkers Prev 2009 18: 541550.

14. Znaor A, Brennan P, Gajalakshmi V et al. Independent and combined effects of tobacco smoking, chewing and alcohol drinking on the risk of oral, pharyngeal and oesophageal cancers in Indian men. Int $\mathbf{J}$ Cancer 2003 105: 681-686.

15. Gupta PC, Warnakulasuriya S. Global epidemiology of areca nut usage. Addiction Biology 2002;7:77-83.

16. Gupta PC, Ray CS. Epidemiology of betel quid usage. Ann Acad Med Singapore 2004 33: 31-36.

17. Li Y, Cheng A, Lee L, Huang Y, Chang J. Multifaceted Mechanisms of Areca Nuts in Oral Carcinogenesis: the Molecular Pathology from Precancerous Condition to Malignant Transformation. Journal of Cancer. 2019;10(17):4054-4062.

18. Furniss CS, McClean MD, Smith JF et al. Human papillomavirus 16 seropositivity is associated with risk of head and neck squamous cell carcinoma, independent of tobacco and alcohol use. Ann Oncol 2009 20: 534-541.

19. Balaram $P$, Nalinakumari KR, Abraham E et al. Human papillomaviruses in 91 oral cancers from Indian betel quid chewers- 
Hindol Das et.al. India as "the oral cancer capital of the world": the rising burden of oral malignancies across the nation.

high prevalence and multiplicity of infections. Int J Cancer 1995 61: 450-454.

20. R. Sankaranarayanan, K. Ramadas, G. Thomas et al., "Effect of screening on oral cancer mortality in Kerala, India: a clusterrandomised controlled trial," The Lancet, vol. 365, no. 9475, pp. 1927-1933, 2005

21. Chaturvedi P. Effective strategies for oral cancer control in India. J Can Res Ther 2012;8, Suppl S2:55-6

22. Mathew B, Sankaranarayanan R, Sunilkumar KB et al. Reproducibility and validity of oral visual inspection by trained health workers in the detection of oral precancer and cancer. Br J Cancer 1997 76: 390-394.

23. Petersen P. Oral cancer prevention and control - The approach of the World Health
Organization. Oral Oncology. 2009;45(45):454-460.

24. Kaur J, Jain DC. Tobacco control policies in India: implementation and challenges. Indian J Public Health 2011 55: 220-227.

25. Jornet P, Garcia F, Berdugo M, Perez F, Lopez A. Mouth self-examination in a population at risk of oral cancer. Australian Dental Journal. 2015;60(1):59-64.

26. Gupta B, Ariyawardana A, Johnson N. The epidemic of oral cancer in India continues unabated: Need for new policy initiatives. Oral Oncology. 2012;48(8):e31-e32.

How to cite this article: Das H, Motghare S. India as "the oral cancer capital of the world": the rising burden of oral malignancies across the nation. International Journal of Science \& Healthcare Research. 2021; 6(2): 99-107. DOI: https://doi.org/10.52403/ijshr.20210419 